\title{
Tristan Vigliano, Humanisme et juste milieu au siècle de Rabelais, Essai de critique illusoire
} Paris, Les Belles Lettres, coll. « Le miroir des humanistes», 2009, $739 \mathrm{p}$.

\author{
Grégoire Holtz \\ Université de Toronto
}

Le livre de Tristan Vigliano s'impose au-delà de son épais volume (plus de 700 pages) par le surplomb et la largeur de vue qu'il pose sur un objet aussi déroutant qu'essentiel pour tout chercheur travaillant sur la littérature de la Renaissance : les représentations, justifications et négociations que les humanistes durent mettre en œuvre pour écrire le juste milieu. Son titre, Humanisme et juste milieu au siècle de Rabelais, rend compte de ses bornes chronologiques (le " premier XVIe siècle » d'avant 1550) et surtout de son ambition, qui est de retracer 
l'histoire d'un concept, l'héritage du juste milieu aristotélicien, dans une époque marquée par les profonds bouleversements religieux et politiques qu'on connaît. En s'inscrivant dans la tradition de l'histoire des idées, T. Vigliano étudie un corpus qu'il situe aux confins de la littérature, de la philosophie et de la morale. On appréciera son choix de ne pas se cantonner aux grands noms du panthéon humaniste (Valla, Érasme, Budé, M. de Navarre, Rabelais, etc.) mais d'intégrer des minores (J. Clichtove, J. Mayr ou P. Tateret), qui viennent compléter et parfaire ce panorama de la vie intellectuelle confrontée à la question épineuse du juste milieu.

Ce travail considérable permet d'abord de mieux mesurer la diversité des différentes lectures de l'Éthique à Nicomaque, dont découle la réflexion sur le juste milieu. Dans la filiation du travail pionnier de $\mathrm{Ch}$. Schmitt, l'ouvrage présente les fortunes du texte d'Aristote qui est lu à travers ses commentateurs explicites, mais qui est aussi connu grâce à la diffusion plus large des idées du Stagirite dans la littérature morale et dans les différents champs du savoir. La première partie revient précisément sur l'Éthique à Nicomaque, en dégageant le ou les sens que prend le juste milieu dans la réflexion d'Aristote, en soulignant les ambiguïtés et les potentialités interprétatives que ce texte stimulera. Appliquée aux notions de justice, de vertu, de prudence et à l'équilibre entre les moyens et les fins, la notion de juste milieu, pensée comme une moyenne entre deux extrêmes, a pu provoquer dès son origine des contresens et des lectures divergentes. En particulier, la difficulté à distinguer les intentions vertueuses des vicieuses (comprises selon une différenciation par degrés), et donc la propension à suivre cyniquement son intérêt personnel en dépit de toute fin morale, 
ouvre la voie à une « lecture opportuniste » du juste milieu, qui expliquera la méfiance qui entoure ce concept à la Renaissance.

Cette présentation du problème chez Aristote donne son orientation aux deux principales parties du livre : d'une part, les «discours classiques" rassemblent des auteurs qui, au XVI siècle, «admettent la difficulté, mais non pas l'impossibilité d'atteindre une moyenne entre les extrêmes » (p. 18); d'autre part, les «voix discordantes » rejettent, essentiellement pour des motifs religieux, mais aussi au nom d'une critique de l'aristotélisme et de la scolastique, la théorie du juste milieu. Dans la première catégorie, sont exposés les réflexions des théologiens et moralistes, mais aussi les choix de certains savants (juristes, médecins et pédagogues) qui tentent de délimiter un possible exercice du juste milieu, parfois au prix de certaines dissymétries, d'acrobaties argumentatives ou encore d'un renouvellement du commentaire d'Aristote (voir par exemple les pages consacrées à la position de Melanchton, qui glose l'Éthique à Nicomaque et redéfinit la sensibilité humaine comme un "juste milieu entre l'épicurisme et le stoïcisme», p. 309). Dans la seconde catégorie sont réunies des réflexions anti-aristotéliciennes qui, de Pétrarque à Valla, de Pic à Budé, rejettent le juste milieu parce que ces auteurs refusent l'autorité du Stagirite et/ou parce qu'ils affirment le primat de la foi sur l'empirisme et l'intellectualisme logique d'Aristote. Les pages consacrées à Érasme et M. de Navarre montrent à quel point l'évangélisme s'est nourri d'un équilibre précaire entre la recherche d'un juste milieu et les "mortifications » que cette difficile recherche engendre. C'est dire à quel point la synthèse précaire entre leur sagesse chrétienne et leur formation philosophique païenne constitue un point d'achoppement particulièrement sensible dans leur pensée. Cette recherche 
chancelante d'une sagesse en équilibre instable prend chez ces deux auteurs la forme de de l'illusion consentie: alors qu'Érasme, dans l'Éloge de la folie, revendique un usage paradoxal de l'erreur, comprise comme une acceptation consentie des pouvoirs de l'illusion (cf. le paradoxe dit du Crétois : «Si Folie exige qu'on maintienne l'illusion, c'est qu'elle n'en est pas dupe elle-même», p. 475), Marguerite, dans son théâtre et sa prose, dessine les contours d'une morale oxymorique et d'une " oscillation illusoire » entre les extrêmes, permettant là encore une réconciliation illusoire entre sagesse divine et folie humaine.

Enfin, la dernière voix discordante, particulièrement singulière, est celle de Rabelais, qui occupe le dernier volet de l'ouvrage. T. Vigliano étudie l'ensemble de son œuvre à travers le prisme du juste milieu, mais il voit aussi dans la geste rabelaisienne l'aboutissement et la radicalisation de l'impossible quête par laquelle les esprits de la Renaissance ont tenté de résoudre l'aporie du juste milieu. Après avoir présenté (parfois de manière caricaturale) le panorama de la bibliographie critique rabelaisienne, l'auteur démontre comment la problématique du juste milieu permet de réconcilier approches formalistes et "évangéliques ». La thèse de l'illusion consentie, que l'auteur prête au pantagruélisme, lui permet de dépasser les limites de chacune des traditions critiques et de voir chez Rabelais un "dialogue entre les extrêmes » de la sagesse et de la folie au nom "d'une pédagogie sciemment et joyeusement impossible » (p. 596-597). En revenant sur des passages allégoriques très célèbres comme le prologue du Gargantua ou la séquence des «Paroles gelées », l'interprétation de T. Vigliano maintient un point d'équilibre précaire qui, sans verser dans un scepticisme 
intégral, refuse de voir dans le cycle rabelaisien la présence d'une vérité révélée.

En pointant la possibilité d'une lecture autotélique, permise par la séduction d'un langage qui ne vaudrait que pour lui-même, l'auteur boucle sa réflexion sur la délicate et sans doute insoluble question des intentions de Rabelais : l'hypothèse de l'autotélisme distinguerait Rabelais d'Érasme et de Marguerite, ruinant le postulat de l'illusion consentie puisque «la polysémie ne serait plus conciliable avec l'évangélisme» (p.674). En rappelant cependant que «Rabelais donne tous les gages d'une piété profonde, même si c'est par l'intermédiaire de ses personnages ", T. Vigliano conclut sur l'ambiguïté irréductible du texte et renvoie le lecteur à sa liberté interprétative, un geste qui reproduit l'appel de Rabelais et de son «Fays ce que voudras ».

Ce résumé ne doit ni occulter l'intérêt essentiel de cet ouvrage, ni minorer la richesse de ses interprétations. On appréciera l'ampleur du corpus traité qui, dans le massif foisonnant de textes abordant la représentation du juste milieu, permet de reconstituer les tendances du débat moral qui agitait les humanistes (l'auteur aurait aussi pu inclure dans sa réflexion les mythes d'un impossible juste milieu, comme ceux d'Icare ou de Phaéton, qui nourrissent les gloses allégoriques des mythographes). On retiendra aussi le choix méthodologique indiqué par le sous-titre "Essai de critique illusoire ». Habile à manier le paradoxe cher aux auteurs qu'il étudie, T. Vigliano met en pratique dans sa réflexion même la stratégie du retournement qui, sans être facile ou vaine, révèle les soubresauts dialectiques, les asymétries et dissymétries de 
raisonnements tentant, souvent en vain, de trouver une voie moyenne entre les extrêmes.

Sans jamais tomber dans le catalogue, l'étude de T. Vigliano montre avec rigueur la fécondité et les équivocités de la notion de juste milieu. Ce faisant, il vient compléter une lacune que de rares études avaient commencé à combler, comme l'ouvrage collectif portant sur l'Éloge de la médiocrité ou encore la passionnante étude sur les "Moyenneurs », Ni Rome ni Genève, du regretté Th. Wanegfellen. Enfin, et c'est le plus important, l'essai de T.Vigliano permet d'apporter une contribution à la compréhension de l'humanisme en réfléchissant sur ses zones d'ombres et sur les limites constitutives de son projet. L'enjeu central de la réflexion revient à discuter les conditions de possibilité d'un accord entre la transmission des sources païennes (dans lesquelles l'aristotélisme occupait une place de choix) et le cadre chrétien de la Révélation qui les accueille. Le juste milieu est d'abord celui-ci : réussir à penser le syncrétisme entre paganisme et christianisme, tenter une synthèse entre des systèmes moraux et des autorités qui présentent de nombreux points de discordance. Dans cette quête vertigineuse du sens autour du juste milieu, on conclura qu'importe moins l'arrivée à une conclusion finale que le parcours et la mobilité de la pensée, bref la saisie dans son mouvement même d'un « sens agile». 